\title{
Intuitionistic fuzzy dimension of an intuitionistic fuzzy vector space
}

\author{
Moumita Chiney $^{1}$ and S. K. Samanta ${ }^{2}$ \\ Department of Mathematics, Visva-Bharati \\ Santiniketan - 731235, West Bengal, India \\ e-mails: moumi.chiney@gmail.com, syamal_123@yahoo.co.in
}

Received: 25 October 2017 Revised: 28 November $2017 \quad$ Accepted: 2 December 2017

Abstract: In the present paper the notion of intuitionistic fuzzy dimension of an intuitionistic fuzzy vector space has been developed with the help of intuitionistic fuzzy basis.

Keywords: Intuitionistic fuzzy sets, Intuitionistic fuzzy vector space, Intuitionistic fuzzy dimension.

2010 Mathematics Subject Classification: 03E72, 15A03.

\section{Introduction}

The notion of intuitionistic fuzzy set (IFS) was introduced by Atanassov [1, 2, 3, 4] as a generalization of Zadeh's fuzzy set [22]. There are situations where IFS theory is more appropriate to dealt with [7]. IFS theory have successfully been applied in knowledge engineering, medical diagnosis, decision making, career determination, etc., [11, 21, 12]. Several researchers have extended various mathematical aspects such as groups, rings, topological spaces, metric spaces, topological groups, topological vector spaces etc. in IFS $[6,10,13,16,17,18,19]$. The notion of fuzzy vector subspaces has been introduced by Katsaras [14] and a notion of fuzzy bases and fuzzy dimension was studied by Shi et al. [20]. We have introduced a notion of intuitionistic fuzzy vector space and intuitionistic fuzzy basis in [9]. As a continuation of our paper [9], here we introduced the notion of intuitionistic fuzzy dimension of an intuitionistic fuzzy vector space with the help of intuitionistic fuzzy basis and studied some of its basic results. 


\section{Preliminaries}

Definition 2.1. [1] Let $X$ be a non-empty set. An intuitionistic fuzzy set (IFS for short) of $X$ is defined as an object having the form $A=\left\{\left\langle x, \mu_{A}(x), v_{A}(x)\right\rangle \mid x \in X\right\}$, where $\mu_{A}: X \rightarrow[0,1]$ and $v_{A}$ : $X \rightarrow[0,1]$ denote the degree of membership (namely $\mu_{A}(x)$ ) and the degree of non-membership (namely $v_{A}(x)$ ) of each element $x \in X$ to the set $A$, respectively, and $0 \leq \mu_{A}(x)+v_{A}(x) \leq 1$ for each $x \in X$. For the sake of simplicity we shall use the symbol $A=\left(\mu_{A}, v_{A}\right)$ for the intuitionistic fuzzy set $A=\left\{\left\langle x, \mu_{A}(x), v_{A}(x)\right\rangle \mid x \in X\right\}$.

In this paper, we use the symbols $a \wedge b=\min \{a, b\}$ and $a \vee b=\max \{a, b\}$.

Definition 2.2. [1] Let $A=\left(\mu_{A}, v_{A}\right)$ and $B=\left(\mu_{B}, v_{B}\right)$ be intuitionistic fuzzy sets of a set $X$. Then

(1) $A \subseteq B$ iff $\mu_{A}(x) \leq \mu_{B}(x)$ and $v_{A}(x) \geq v_{B}(x)$ for all $x \in X$.

(2) $A=B$ iff $A \subseteq B$ and $B \subseteq A$.

(3) $A^{c}=\left\{\left\langle x, v_{A}(x), \mu_{A}(x)\right\rangle \mid x \in X\right\}$

(4) $A \cap B=\left\{\left\langle x, \mu_{A}(x) \wedge \mu_{B}(x), v_{A}(x) \vee v_{B}(x)\right\rangle \mid x \in X\right\}$.

(5) $A \cup B=\left\{\left\langle x, \mu_{A}(x) \vee \mu_{B}(x), v_{A}(x) \wedge v_{B}(x)\right\rangle \mid x \in X\right\}$.

(6) $\square A=\left\{\left\langle x, \mu_{A}(x), 1-\mu_{A}(x)\right\rangle \mid x \in X\right\}, \diamond A=\left\{\left\langle x, 1-v_{A}(x), v_{A}(x)\right\rangle \mid x \in X\right\}$.

Definition 2.3. [4] Let $A$ be an IFS in a set $X$. Then for $\lambda, \xi \in[0,1]$ with $\lambda+\xi \leq 1$, the set $A^{[\lambda, \xi]}=\left\{x \in X: \mu_{A}(x) \geq \lambda\right.$ and $\left.v_{A}(x) \leq \xi\right\}$ is called $(\lambda, \xi)$-level subset of $A$.

Proposition 2.4. [4] Let $A$ be an IFS in a set $X$ and $\left(\lambda_{1}, \xi_{1}\right),\left(\lambda_{2}, \xi_{2}\right) \in \operatorname{Im}(A)$. If $\lambda_{1} \leq \lambda_{2}$ and $\xi_{1} \geq \xi_{2}$, then $A^{\left[\lambda_{1}, \xi_{1}\right]} \supseteq A^{\left[\lambda_{2}, \xi_{2}\right]}$.

Definition 2.5. [15, 5] Let $X$ be a vector space over the field $K$, the field of real and complex numbers, $\alpha \in K, A=\left(\mu_{A}, v_{A}\right)$ and $B=\left(\mu_{B}, v_{B}\right)$ be two intuitionistic fuzzy sets of $X$. Then

(1) the sum of $A$ and $B$ is defined to be the intuitionistic fuzzy set $A+B=\left(\mu_{A}+\mu_{B}, v_{A}+v_{B}\right)$ of $X$ given by

$$
\begin{aligned}
& \mu_{A+B}(x)= \begin{cases}\sup _{x=a+b}\left\{\mu_{A}(a) \wedge \mu_{B}(b)\right\} & \text { if } x=a+b \\
0 & \text { otherwise, }\end{cases} \\
& v_{A+B}(x)= \begin{cases}\inf _{x=a+b}\left\{v_{A}(a) \vee v_{B}(b)\right\} & \text { if } x=a+b \\
1 & \text { otherwise. }\end{cases}
\end{aligned}
$$

(2) $\alpha A$ is defined to be the IFS $\alpha A=\left(\mu_{\alpha A}, v_{\alpha A}\right)$ of $X$, where

$$
\mu_{\alpha A}(x)= \begin{cases}\mu_{A}\left(\alpha^{-1} x\right) & \text { if } \alpha \neq 0 \\ \sup _{y \in X} \mu_{A}(y) & \text { if } \alpha=0, x=\theta \\ 0 & \text { if } \alpha=0, x \neq \theta\end{cases}
$$




$$
v_{\alpha A}(x)= \begin{cases}v_{A}\left(\alpha^{-1} x\right) & \text { if } \alpha \neq 0 \\ \inf _{y \in X} v_{A}(y) & \text { if } \alpha=0, x=\theta \\ 1 & \text { if } \alpha=0, x \neq \theta .\end{cases}
$$

Proposition 2.6. [9] Let $A, A_{1}, \ldots, A_{n}$ be intuitionistic fuzzy sets in a vector space $X$ and $\lambda_{1}, \ldots, \lambda_{n}$ be scalars. Then the following assertions are equivalent:

(1) $\lambda_{1} A_{1}+\lambda_{2} A_{2}+\cdots+\lambda_{n} A_{n} \subseteq A$.

(2) For all $x_{1}, x_{2}, \ldots, x_{n}$ in $X$, we have

$$
\begin{aligned}
& \mu_{A}\left(\lambda_{1} x_{1}+\lambda_{2} x_{2}+\cdots+\lambda_{n} x_{n}\right) \geq \min \left\{\mu_{A_{1}}\left(x_{1}\right), \mu_{A_{2}}\left(x_{2}\right), \ldots, \mu_{A_{n}}\left(x_{n}\right)\right\} \text { and } v_{A}\left(\lambda_{1} x_{1}+\lambda_{2} x_{2}+\right. \\
& \left.\cdots+\lambda_{n} x_{n}\right) \leq \max \left\{v_{A_{1}}\left(x_{1}\right), v_{A_{2}}\left(x_{2}\right), \ldots, v_{A_{n}}\left(x_{n}\right)\right\} .
\end{aligned}
$$

Definition 2.7. [9] An IFS $V=\left(\mu_{V}, v_{V}\right)$ of a vector space $X$ over the field $K$ is said to be intuitionistic fuzzy vector space over $X$ if

(i) $V+V \subseteq V$

(ii) $\alpha V \subseteq V$, for every scalar $\alpha$.

We denote the set of all intuitionistic fuzzy vector spaces over a vector space X by IFVS $(X)$.

Remark 2.8. [9] Let $X$ be a vector space.

(1) If $\mu_{V}$ is a fuzzy subspace of $X$, then $V=\left(\mu_{V}, \mu_{V}^{c}\right) \in \operatorname{IFVS}(X)$.

(2) If $V \in \operatorname{IFVS}(X)$, then $\mu_{V}$ and $v_{V}^{c}$ are fuzzy vector subspace of $X$.

(3) If $V \in \operatorname{IFVS}(X)$, then $\square V, \diamond V \in \operatorname{IFVS}(X)$.

Lemma 2.9. [9] Let $V$ be an intuitionistic fuzzy set in a vector space $X$. Then, the following are equivalent:

(1) $V$ is an intuitionistic fuzzy vector space over X.

(2) For all scalars $\alpha, \beta$, we have $\alpha V+\beta V \subseteq V$.

(3) For all scalars $\alpha, \beta$ and for all $x, y \in X$, we have

$$
\left.\mu_{V}(\alpha x+\beta y) \geq \mu_{V}(x) \wedge \mu_{V}(y)\right\} \text { and } v_{V}(\alpha x+\beta y) \leq v_{V}(x) \vee v_{V}(y) .
$$

Remark 2.10. [9] Our definition of intuitionistic fuzzy vector space is equivalent to the definition of intuitionistic fuzzy subspace of [19] and [8].

Proposition 2.11. [8] If $V, W \in \operatorname{IFVS}(X)$, then $V+W \in \operatorname{IFVS}(X)$.

Proposition 2.12. [9] If $V \in I F V S(X) \alpha \in K$, then $\alpha V \in I F V S(X)$.

Proposition 2.13. [8] If $\left\{V_{i}\right\}_{i \in I} \in \operatorname{IFVS}(X)$, then $\bigcap_{i \in I} V_{i} \in I F V S(X)$. 
Proposition 2.14. [9] Let $V \in I F V S(X)$. Then $\mu_{V}(\theta) \geq \mu_{V}(x)$ and $v_{V}(\theta) \leq v_{V}(x), \forall x \in X$.

Proposition 2.15. [9] Let $V \in \operatorname{IFVS}(X)$. Then for each $(\lambda, \xi) \in[0,1] \times[0,1]$ with $\lambda+\xi \leq 1, \lambda \leq$ $\mu_{V}(\theta)$ and $\xi \geq v_{V}(\theta), V^{[\lambda, \xi]}$ is a subspace of the vector space $X$,

Definition 2.16. [9] For any $(a, b),(c, d) \in[0,1] \times[0,1]$ with $a+b \leq 1, c+d \leq 1$, we say that:

(1) $(a, b) \geq(c, d)$ if $a \geq b$ and $c \leq d$.

(2) $(a, b) \leq(c, d)$ if $a \leq b$ and $c \geq d$.

(3) $(a, b)>(c, d)$ if $a>b$ and $c \leq d$ or if $a \geq b$ and $c<d$.

(4) $(a, b)<(c, d)$ if $a<b$ and $c \geq d$ or if $a \leq b$ and $c>d$.

(5) $(a, b)=(c, d)$ if $a=b$ and $c=d$.

Proposition 2.17. [9] Let $V \in I F V S(X)$ with $\operatorname{dim} X=m$. Then $\operatorname{Im}(V)$ contains at most $m+1$ points of $[0,1] \times[0,1]$.

Definition 2.18. [9] Let $V=\left(\mu_{V}, v_{V}\right) \in \operatorname{IFVS}(X)$. Then for any $\lambda \in \mu_{V}(X), \xi \in v_{V}(X)$ we define

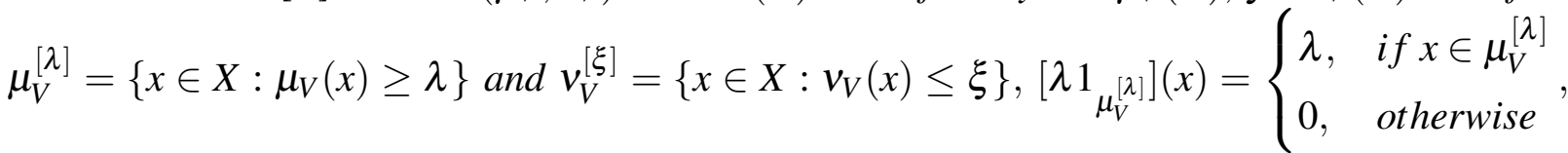
$\left[\xi 1_{\left.v_{V}^{[\xi]}\right]}\right](x)=\left\{\begin{array}{ll}\xi, & \text { if } x \in v_{V}^{[\xi]} \\ 1, & \text { otherwise }\end{array}\right.$.

Theorem 2.19. [9] (Representation Theorem) Let $V \in \operatorname{IFVS}(X)$ with $\operatorname{dim} X=m$ and $\operatorname{Im}(V)=$ $\left\{\left(\lambda_{0}, \xi_{0}\right),\left(\lambda_{1}, \xi_{1}\right), \ldots\left(\lambda_{k}, \xi_{k}\right)\right\}, k \leq m$ such that $(1,0) \geq\left(\lambda_{0}, \xi_{0}\right)>\left(\lambda_{1}, \xi_{1}\right)>\ldots>\left(\lambda_{k}, \xi_{k}\right) \geq(0,1)$. Then there exists nested collection of subspaces of $X$ as $\{\theta\} \subseteq V^{\left[\lambda_{0}, \xi_{0}\right]} \varsubsetneqq V^{\left[\lambda_{1}, \xi_{1}\right]} \varsubsetneqq \ldots \varsubsetneqq V^{\left[\lambda_{k}, \xi_{k}\right]}=$ $X$ such that $\mu_{V}=\lambda_{0} 1_{\mu_{V}^{\left[\lambda_{0}\right]}} \vee \lambda_{1} 1_{\mu_{V}^{\left[\lambda_{1}\right]}} \vee \ldots \vee \lambda_{k} 1_{\mu_{V}^{\left[\lambda_{k}\right]}}$ and $v_{V}=\xi_{0} 1_{v_{V}^{\left[\xi_{0}\right]}} \wedge \xi_{1^{\prime}} 1_{v_{V}^{\left[\xi_{1}\right]}} \wedge \ldots \wedge \xi_{k} 1_{v_{V}^{\left[\xi_{k}\right]}}$. Also,

(1) If $(\zeta, \rho),(\eta, \sigma) \in\left(\lambda_{i+1}, \lambda_{i}\right] \times\left[\xi_{i}, \xi_{i+1}\right)$ with $\zeta+\rho \leq 1, \eta+\sigma \leq 1$, then $V^{[\zeta, \rho]}=V^{[\eta, \sigma]}=$ $V^{\left[\lambda_{i}, \xi_{i}\right]}$.

(2) If $(\zeta, \rho) \in\left(\lambda_{i+1}, \lambda_{i}\right] \times\left[\xi_{i}, \xi_{i+1}\right),(\eta, \sigma) \in\left(\lambda_{i}, \lambda_{i-1}\right] \times\left[\xi_{i-1}, \xi_{i}\right)$ with $\zeta+\rho \leq 1, \eta+\sigma \leq 1$, then $V^{[\zeta, \rho]} \supsetneqq V^{[\eta, \sigma]}$.

Definition 2.20. [9] Let $V \in I F V S(X)$ with $\operatorname{dim} X=m$. Consider Theorem 2.19. Let $B_{V_{i}}$ be the basis of $V^{\left[\lambda_{i}, \xi_{i}\right]}, i=0,1, . ., k$ such that

$$
B_{V_{0}} \varsubsetneqq B_{V_{1}} \varsubsetneqq \cdots \varsubsetneqq B_{V_{k}} .
$$

If $V^{\left(\lambda_{0}, \xi_{0}\right)}=\{\theta\}$, we start with $V^{\left(\lambda_{1}, \xi_{1}\right)}$.

Define a map $\mathbb{B}$ from $X$ to $[0,1] \times[0,1]$ by

$\mu_{\mathbb{B}}(x)=\left\{\begin{array}{l}\vee\left\{\lambda_{i}: x \in B_{V_{i}}\right\} \\ 0, \text { otherwise }\end{array} \quad\right.$ and $v_{\mathbb{B}}(x)=\left\{\begin{array}{l}\wedge\left\{\xi_{i}: x \in B_{V_{i}}\right\} \\ 1, \text { otherwise }\end{array}\right.$. 
Let $\mu_{\mathbb{B}}(x)=\lambda_{j}$. Then $x \in B_{V_{j}}$ and $x \notin B_{V_{j-1}}$ i.e. $x \in V^{\left[\lambda_{j}, \xi_{j}\right]}$ and $x \notin V^{\left[\lambda_{j-1}, \xi_{j-1}\right]}$. Thus $\mu_{V}(x) \geq \lambda_{j}$ and $v_{V}(x) \leq \xi_{j}$. If $\mu_{V}(x)>\lambda_{j}$, then $\mu_{V}(x)=\lambda_{l}$ for some $l<j$. Then $x \in V^{\left[\lambda_{l}, \xi_{l}\right]}$ and $\mu_{(B)}(x)=\lambda_{l}$, which is a contradiction. Therefore $\mu_{V}(x)=\lambda_{j}$. Then $v_{V}(x)=\xi_{j}$ i.e. $v_{\mathbb{B}}(x)=\xi_{j}$. Therefore $\mathbb{B}$ is an intuitionistic fuzzy set and it is called intuitionistic fuzzy basis of $V$ corresponding to $(*)$.

Proposition 2.21. [9] Let $\mathbb{B}$ be an intuitionistic fuzzy basis of $V$ corresponding to $(*)$ of Definition 2.20. Then

(1) If $(\zeta, \rho),(\eta, \sigma) \in\left(\lambda_{i+1}, \lambda_{i}\right] \times\left[\xi_{i}, \xi_{i+1}\right)$ with $\zeta+\rho \leq 1, \eta+\sigma \leq 1$, then $\mathbb{B}^{[\zeta, \rho]}=\mathbb{B}^{[\eta, \sigma]}=$ $B_{V_{i}}$.

(2) If $(\zeta, \rho) \in\left(\lambda_{i+1}, \lambda_{i}\right] \times\left[\xi_{i}, \xi_{i+1}\right),(\eta, \sigma) \in\left(\lambda_{i}, \lambda_{i-1}\right] \times\left[\xi_{i-1}, \xi_{i}\right)$ with $\zeta+\rho \leq 1, \eta+\sigma \leq 1$, then $\mathbb{B}^{[\zeta, \rho]} \supsetneqq \mathbb{B}^{[\eta, \sigma]}$.

(3) $\mathbb{B}^{[\lambda, \xi]}$ is a basis of $V^{[\lambda, \xi]}$ for $\lambda \in(0,1], \xi \in[0,1)$ with $\lambda+\xi \leq 1$.

Proposition 2.22. Let $\mathbb{B}$ be an intuitionistic fuzzy basis of $V$ corresponding to $(*)$ of Definition 2.20. Then $\mu_{\mathbb{B}}^{\left[\lambda_{i}\right]}=B_{V_{i}}=v_{\mathbb{B}}^{\left[\xi_{i}\right]}$, for $i=0,1,2, . ., k$.

Proof. Let $x \in \mu_{\mathbb{B}}^{\left[\lambda_{i}\right]} \Rightarrow \mu_{\mathbb{B}}(x) \geq \lambda_{i}$. Let $\mu_{\mathbb{B}}(x)=\lambda_{j} \Rightarrow x \in B_{V_{j}} \subset B_{V_{i}}$.

Thus $\mu_{\mathbb{B}}^{\left[\lambda_{i}\right]} \subseteq B_{V_{i}}$. Conversely, let $x \in B_{V_{i}} \Rightarrow \mu_{V}(x) \geq \lambda_{i}$.

Let $\mu_{V}(x)=\lambda_{j}$. If $\lambda_{j}>\lambda_{i}$, then $\mu_{\mathbb{B}}(x)=\lambda_{j}$.

If $\lambda_{j}=\lambda_{i}$, then $\mu_{\mathbb{B}}(x) \geq \lambda_{i}$. Therefore, in any case $x \in \mu_{\mathbb{B}}^{\left[\lambda_{i}\right]}$.

Thus $B_{V_{i}} \subseteq \mu_{\mathbb{B}}^{\left[\lambda_{i}\right]}$. Hence $\mu_{\mathbb{B}}^{\left[\lambda_{i}\right]}=B_{V_{i}}$.

Similarly, it can be proved that $B_{V_{i}}=v_{\mathbb{B}}^{\left[\xi_{i}\right]}$.

Proposition 2.23. Let $V \in \operatorname{IFVS}(X)$ with $\operatorname{dim} X=m$ and $\operatorname{Im}(V)=\left\{\left(\lambda_{0}, \xi_{0}\right),\left(\lambda_{1}, \xi_{1}\right), \ldots\left(\lambda_{k}, \xi_{k}\right)\right\}, k \leq$ $m$ such that $(1,0) \geq\left(\lambda_{0}, \xi_{0}\right)>\left(\lambda_{1}, \xi_{1}\right)>\ldots>\left(\lambda_{k}, \xi_{k}\right) \geq(0,1)$. Then for $i=0,1, . ., k, V^{\left[\lambda_{i}, \xi_{i}\right]}=$ $\mu_{V}^{\left[\lambda_{i}\right]}=v_{V}^{\left[\xi_{i}\right]}$.

Proof. Obviously, $V^{\left[\lambda_{i}, \xi_{i}\right]} \subseteq \mu_{V}^{\left[\lambda_{i}\right]}$.

Let $x \in \mu_{V}^{\left[\lambda_{i}\right]}$.

$\Rightarrow \mu_{V}(x) \geq \lambda_{i}$.

Let $\mu_{V}(x)=\lambda_{j}$. Then $v_{V}(x)=\xi_{j}$.

$\Rightarrow x \in V^{\left[\lambda_{j}, \xi_{j}\right]}$

$\Rightarrow x \in V^{\left[\lambda_{i}, \xi_{i}\right]}\left[\right.$ as either $\left(\lambda_{j}, \xi_{j}\right)=\left(\lambda_{i}, \xi_{i}\right)$ or $\left.\left(\lambda_{j}, \xi_{j}\right)>\left(\lambda_{i}, \xi_{i}\right)\right]$.

Thus $\mu_{\mathbb{B}}^{\left[\lambda_{i}\right]} \subseteq V^{\left[\lambda_{i}, \xi_{i}\right]}$. Therefore $V^{\left[\lambda_{i}, \xi_{i}\right]}=\mu_{V}^{\left[\lambda_{i}\right]}$.

Similarly we have $V^{\left[\lambda_{i}, \xi_{i}\right]}=v_{V}^{\left[\xi_{i}\right]}$.

Proposition 2.24. Let $\mathbb{B}$ be an intuitionistic fuzzy basis of $V$ corresponding to $(*)$ of Definition 2.20. Then $\left|\mu_{\mathbb{B}}^{\left[\lambda_{i}\right]}\right|=\operatorname{dim}\left(\mu_{V}^{\left[\lambda_{i}\right]}\right)$ and $\left|v_{\mathbb{B}}^{\left[\xi_{i}\right]}\right|=\operatorname{dim}\left(v_{V}^{\left[\xi_{i}\right]}\right)$, for $i=0,1,2, . ., k$.

Proof. $\left|\mu_{\mathbb{B}}^{\left[\lambda_{i}\right]}\right|=\left|B_{V_{i}}\right|=\operatorname{dim}\left(V^{\left[\lambda_{i}, \xi_{i}\right]}\right)=\operatorname{dim}\left(\mu_{V}^{\left[\lambda_{i}\right]}\right)$ [By Proposition 2.22 and 2.23].

The rest part is similar. 


\section{Intuitionistic fuzzy dimension}

Definition 3.1. Let $A$ be an intuitionistic fuzzy set over $X$. Define a map $|A|: \mathbb{N} \rightarrow[0,1] \times[0,1]$ such that $\forall n \in \mathbb{N}, \mu_{|A|}(n)=\vee\left\{a:(a, b) \in[0,1] \times[0,1] \backslash\{(0,1)\}\right.$ with $a+b \leq 1$ and $\left.\left|A^{[a, b]}\right| \geq n\right\}$ and $v_{|A|}(n)=\wedge\left\{b:(a, b) \in[0,1] \times[0,1] \backslash\{(0,1)\}\right.$ with $a+b \leq 1$ and $\left.\left|A^{[a, b]}\right| \geq n\right\}$. Then $|A|$ is an intuitionistic fuzzy set over $\mathbb{N}$, which is called the cardinality of $A$.

Definition 3.2. For two IFS $A, B$ over $X$, the addition $|A|+|B|$ of $|A|$ and $|B|$ is defined as follows: for any $n \in \mathbb{N}, \mu_{(|A|+|B|)}(n)=\vee_{k+l=n}\left(\mu_{|A|}(k) \wedge \mu_{|B|}(l)\right)$ and $v_{(|A|+|B|)}(n)=$ $\wedge_{k+l=n}\left(v_{|A|}(k) \vee v_{|B|}(l)\right)$.

Proposition 3.3. For two IFS $|A|,|B|$ over $\mathbb{N}$ and for any $(a, b) \in[0,1] \times[0,1]$ with $a+b \leq 1$, $\mu_{(|A|+|B|)}^{[a]}=\mu_{|A|}^{[a]}+\mu_{|B|}^{[a]}$ and $v_{(|A|+|B|)}^{[b]}=v_{|A|}^{[b]}+v_{|B|}^{[b]}$.

Proof. First we prove that $\mu_{(|A|+|B|)}^{[a]} \subseteq \mu_{|A|}^{[a]}+\mu_{|B|}^{[a]}$.

Let $n \in \mu_{(|A|+|B|)}^{[a]}$. Then $\mu_{(|A|+|B|)}(n)=\vee_{k+l=n}\left(\mu_{|A|}(k) \wedge \mu_{|B|}(l)\right) \geq a$.

Hence there exist $k, l$ such that $n=k+l$ and $\mu_{|A|}(k) \wedge \mu_{|B|}(l) \geq a$. Then $k \in \mu_{|A|}^{[a]}$ and $l \in \mu_{|B|}^{[a]}$, i.e., $n=k+l \in \mu_{|A|}^{[a]}+\mu_{|B|}^{[a]}$. Similarly, it can be proved that $v_{(|A|+|B|)}^{[b]} \subseteq v_{|A|}^{[a]}+v_{|B|}^{[b]}$.

Conversely suppose that $n \in \mu_{|A|}^{[a]}+\mu_{|B|}^{[a]}$.

Then there exist $k, l$ such that $n=k+l$ with $k \in \mu_{|A|}^{[a]}, l \in \mu_{|B|}^{[a]}$. Then $\left(\mu_{|A|}\right)(k) \geq a,\left(\mu_{|B|}\right)(l) \geq a$. Therefore $\mu_{(|A|+|B|)}(n)=\vee_{k+l=n}\left(\mu_{|A|}(k) \wedge \mu_{|B|}(l)\right) \geq a$. Thus $n \in \mu_{(|A|+|B|)}^{[a]}$.

Hence $\mu_{|A|}^{[a]}+\mu_{|B|}^{[a]} \subseteq \mu_{(|A|+|B|)}^{[a]}$.

Similarly, we have $v_{|A|}^{[a]}+v_{|B|}^{[b]} \subseteq v_{(|A|+|B|)}^{[b]}$. Hence proved.

Definition 3.4. Let $V \in I F V S(X)$ with an intuitionistic fuzzy basis $\mathbb{B}$. Define $\operatorname{dim}(V)=|\mathbb{B}|$. Then $\operatorname{dim}(V)$ is called intuitionistic fuzzy dimension of $V$.

Proposition 3.5. Let $\mathbb{B}$ and $\mathbb{B}^{\prime}$ be two intuitionistic fuzzy bases of an intuitionistic fuzzy vector space $V \in \operatorname{IFVS}(X)$. Then $|\mathbb{B}|=\left|\mathbb{B}^{\prime}\right|$.

Proof. By Proposition 2.21, $\mathbb{B}^{[a, b]}$ and $\mathbb{B}^{{ }^{[a, b]}}$ are bases of $V^{[a, b]}$ for $a \in(0,1], b \in[0,1)$ with $a+b \leq 1$. Then $\left|\mathbb{B}^{[a, b]}\right|=\left|\mathbb{B}^{\prime[a, b]}\right|$.

Hence for any $n \in \mathbb{N}$,

$\mu_{|\mathbb{B}|}(n)=\vee\left\{a:(a, b) \in[0,1] \times[0,1] \backslash\{(0,1)\}\right.$ with $a+b \leq 1$ and $\left.\left|\mathbb{B}^{[a, b]}\right| \geq n\right\}$

$=\vee\left\{a:(a, b) \in[0,1] \times[0,1] \backslash\{(0,1)\}\right.$ with $a+b \leq 1$ and $\left.\left|\mathbb{B}^{\prime[a, b]}\right| \geq n\right\}$

$=\mu_{\left|\mathbb{B}^{\prime}\right|}(n)$. Similarly, for any $n \in \mathbb{N}, v_{|\mathbb{B}|}(n)=v_{\left|\mathbb{B}^{\prime}\right|}(n)$. Hence proved.

Remark 3.6. Intuitionistic fuzzy dimension of an intuitionistic fuzzy vector space is independent of intuitionistic fuzzy basis.

Proposition 3.7. Let $X$ be a vector space with $\operatorname{dim} X=m$ and $V \in \operatorname{IFVS}(X)$. Then for any $(a, b) \in$ $[0,1] \times[0,1] \backslash\{(0,1)\}$ with $a+b \leq 1$ and $n \in \mathbb{N}, n \in \mu_{\operatorname{dim}(V)}^{[a]} \Leftrightarrow n \leq \operatorname{dim}\left(\mu_{V}^{[a]}\right)$ and $n \in v_{\operatorname{dim}(V)}^{[b]} \Leftrightarrow$ $n \leq \operatorname{dim}\left(v_{V}^{[b]}\right)$. 
Proof. Suppose that $\operatorname{Im}(V)=\left\{\left(\lambda_{0}, \xi_{0}\right),\left(\lambda_{1}, \xi_{1}\right), \ldots\left(\lambda_{k}, \xi_{k}\right)\right\}, k \leq m$ such that $(1,0) \geq\left(\lambda_{0}, \xi_{0}\right)>$ $\left(\lambda_{1}, \xi_{1}\right)>\ldots>\left(\lambda_{k}, \xi_{k}\right) \geq(0,1)$. Then there exists a nested collection of subspaces of $X$ as $\{\theta\} \subseteq$ $V^{\left[\lambda_{0}, \xi_{0}\right]} \varsubsetneqq V^{\left[\lambda_{1}, \xi_{1}\right]} \varsubsetneqq \ldots \varsubsetneqq V^{\left[\lambda_{k}, \xi_{k}\right]}=X$

Let $B_{V_{i}}$ be the basis of $V^{\left[\lambda_{i}, \xi_{i}\right]}, i=0,1, . ., k$ such that $\left.B_{V_{0}} \varsubsetneqq B_{V_{1}} \varsubsetneqq \ldots \ldots \varsubsetneqq B_{V_{k}} \ldots \ldots .{ }^{*}\right)$.

Let $\mathbb{B}$ be an intuitionistic fuzzy basis corresponding to $(*)$ defined as in Definition 2.20. Let $n \in \mu_{\operatorname{dim}(V)}^{[a]} \Rightarrow \mu_{\operatorname{dim}(V)}(n) \geq a \Rightarrow \vee\left\{c:(c, d) \in(0,1] \times[0,1)\right.$ with $c+d \leq 1$ and $\left.\left|\mathbb{B}^{[c, d]}\right| \geq n\right\} \geq a$. Then there exists $(c, d) \in[0,1] \times[0,1] \backslash\{(0,1)\}$ with $c+d \leq 1$ such that $c \geq a$ and $\left|\mathbb{B}^{[c, d]}\right| \geq n$. Now $\operatorname{dim}\left(\mu_{V}^{[a]}\right)=\left|\mu_{\mathbb{B}}^{[a]}\right| \geq\left|\mu_{\mathbb{B}}^{[c]}\right| \geq\left|\mathbb{B}^{[c, d]}\right| \geq n$.

Conversely suppose that $n \leq \operatorname{dim}\left(\mu_{V}^{[a]}\right)=\left|\mu_{\mathbb{B}}^{[a]}\right|$. Now $a \in\left(\lambda_{i+1}, \lambda_{i}\right]$, for some $i$. Hence $\left|\mu_{\mathbb{B}}^{[a]}\right|=\mid$ $\mu_{\mathbb{B}}^{\left[\lambda_{i}\right]}|=| B_{V_{i}}|=| \mathbb{B}^{\left[\lambda_{i}, \xi_{i}\right]} \mid$. Then $\mu_{\operatorname{dim}(V)}(n)=\vee\{c:(c, d) \in[0,1] \times[0,1] \backslash\{(0,1)\}$ with $c+d \leq$ 1 and $\left.\left|\mathbb{B}^{[c, d]}\right| \geq n\right\} \geq \lambda_{i} \geq a \Rightarrow n \in \mu_{\operatorname{dim}(V)}^{[a]}$. Hence $n \in \mu_{(\operatorname{dim}(V))}^{[a]} \Leftrightarrow n \leq \operatorname{dim}\left(\mu_{V}^{[a]}\right)$.

Similarly it can be proved that $n \in v_{\operatorname{dim}(V)}^{[b]} \Leftrightarrow n \leq \operatorname{dim}\left(v_{V}^{[b]}\right)$.

Proposition 3.8. Let $X$ be a vector space with $\operatorname{dim} X=m$ and $V_{1}, V_{2} \in \operatorname{IFVS}(X)$. Then we have the following results:

(1) For all $(a, b) \in[0,1] \times[0,1]$ with $a+b \leq 1, \mu_{V_{1} \cap V_{2}}^{[a]}=\mu_{V_{1}}^{[a]} \cap \mu_{V_{2}}^{[a]}$ and $v_{V_{1} \cap V_{2}}^{[b]}=v_{V_{1}}^{[b]} \cap v_{V_{2}}^{[b]}$.

(2) For all $(a, b) \in[0,1] \times[0,1]$ with $a+b \leq 1, \mu_{\left(V_{1}+V_{2}\right)}^{[a]}=\mu_{V_{1}}^{[a]}+\mu_{V_{2}}^{[a]}$ and $v_{\left(V_{1}+V_{2}\right)}^{[b]}=v_{V_{1}}^{[b]}+v_{V_{2}}^{[b]}$.

Proof. We only give the proof of (2). For any $(a, b) \in[0,1] \times[0,1]$ with $a+b \leq 1$, we have $x \in \mu_{\left(V_{1}+V_{2}\right)}^{[a]} \Leftrightarrow \sup _{x=x_{1}+x_{2}}\left\{\mu_{V_{1}}\left(x_{1}\right) \wedge \mu_{V_{2}}\left(x_{2}\right)\right\} \geq a$

$\Leftrightarrow$ there exist $x_{1}, x_{2}$ such that $x_{1}+x_{2}=x$ and $\mu_{V_{1}}\left(x_{1}\right) \wedge \mu_{V_{2}}\left(x_{2}\right) \geq a$

$\Leftrightarrow$ there exist $x_{1}, x_{2}$ such that $x_{1}+x_{2}=x$ and $x_{1} \in \mu_{V_{1}}^{[a]}$ and $x_{2} \in \mu_{V_{2}}^{[a]}$.

Similarly it can be proved that $v_{\left(V_{1}+V_{2}\right)}^{[b]}=v_{V_{1}}^{[b]}+v_{V_{2}}^{[b]}$.

Proposition 3.9. Let $X$ be a vector space with $\operatorname{dim} X=m$ and $V_{1}, V_{2} \in \operatorname{IFVS}(X)$. Then $\operatorname{dim}\left(V_{1}+V_{2}\right)+\operatorname{dim}\left(V_{1} \cap V_{2}\right)=\operatorname{dim}\left(V_{1}\right)+\operatorname{dim}\left(V_{2}\right)$.

Proof. For any $(a, b) \in[0,1] \times[0,1]$ with $a+b \leq 1$, let $n \in \mu_{\left(\operatorname{dim}\left(V_{1}+V_{2}\right)+\operatorname{dim}\left(V_{1} \cap V_{2}\right)\right)}^{[a]}$. Then there exist $k, l$ such that $n=k+l$ and $k \in \mu_{\left(\operatorname{dim}\left(V_{1}+V_{2}\right)\right)}^{[a]}$ and $l \in \mu_{\left(\operatorname{dim}\left(V_{1} \cap V_{2}\right)\right)}^{[a]}$. Then by Proposition 3.7, $k \leq \operatorname{dim}\left(\mu_{\left(V_{1}+V_{2}\right)}^{[a]}\right)=\operatorname{dim}\left(\mu_{\left(V_{1}\right)}^{[a]}+\mu_{\left(V_{2}\right)}^{[a]}\right)$ and $l \leq \operatorname{dim}\left(\mu_{\left(V_{1} \cap V_{2}\right)}^{[a]}\right)=\operatorname{dim}\left(\mu_{\left(V_{1}\right)}^{[a]} \cap \mu_{\left(V_{2}\right)}^{[a]}\right)$. Thus $n \leq \operatorname{dim}\left(\mu_{\left(V_{1}\right)}^{[a]}+\mu_{\left(V_{2}\right)}^{[a]}\right)+\operatorname{dim}\left(\mu_{\left(V_{1}\right)}^{[a]} \cap \mu_{\left(V_{2}\right)}^{[a]}\right)=\operatorname{dim}\left(\mu_{\left(V_{1}\right)}^{[a]}\right)+\operatorname{dim}\left(\mu_{\left(V_{2}\right)}^{[a]}\right)$.

Then there exist $k^{\prime}$ and $l^{\prime}$ such that $n=k^{\prime}+l^{\prime}$ and $k^{\prime} \leq \operatorname{dim}\left(\mu_{\left(V_{1}\right)}^{[a]}\right)$ and $l^{\prime} \leq \operatorname{dim}\left(\mu_{\left(V_{2}\right)}^{[a]}\right)$. Now by Proposition 3.7, $k^{\prime} \in \mu_{\operatorname{dim}\left(V_{1}\right)}^{[a]}$ and $l^{\prime} \in \mu_{\operatorname{dim}\left(V_{2}\right)}^{[a]}$. Therefore $n=k^{\prime}+l^{\prime} \in \mu_{\operatorname{dim}\left(V_{1}\right)}^{[a]}+\mu_{\operatorname{dim}\left(V_{2}\right)}^{[a]}=$ $\mu_{\left(\operatorname{dim}\left(V_{1}\right)+\operatorname{dim}\left(V_{2}\right)\right)}^{[a]}$. Hence $\mu_{\left(\operatorname{dim}\left(V_{1}+V_{2}\right)+\operatorname{dim}\left(V_{1} \cap V_{2}\right)\right)}^{[a]} \subseteq \mu_{\left(\operatorname{dim}\left(V_{1}\right)+\operatorname{dim}\left(V_{2}\right)\right)}^{[a]}$.

Similarly, $v_{\left(\operatorname{dim}\left(V_{1}+V_{2}\right)+\operatorname{dim}\left(V_{1} \cap V_{2}\right)\right)}^{[b]} \boldsymbol{v}_{\left(\operatorname{dim}\left(V_{1}\right)+\operatorname{dim}\left(V_{2}\right)\right)}^{[b]}$.

Also, it can be proved that for any $(a, b) \in[0,1] \times[0,1]$ with $a+b \leq 1, \mu_{\left(\operatorname{dim}\left(V_{1}\right)+\left(\operatorname{dim}\left(V_{2}\right)\right.\right.}^{[a]} \subseteq$ $\mu_{\left(\operatorname{dim}\left(V_{1}+V_{2}\right)+\operatorname{dim}\left(V_{1} \cap V_{2}\right)\right)}^{[a]}$ and $v_{\left(\operatorname{dim}\left(V_{1}\right)+\left(\operatorname{dim}\left(V_{2}\right)\right.\right.}^{[b]} \subseteq v_{\left(\operatorname{dim}\left(V_{1}+V_{2}\right)+\operatorname{dim}\left(V_{1} \cap V_{2}\right)\right)}^{[b]}$. Thus for any $(a, b) \in$ $[0,1] \times[0,1]$ with $a+b \leq 1, \mu_{\left(\operatorname{dim}\left(V_{1}\right)+\left(\operatorname{dim}\left(V_{2}\right)\right.\right.}^{[a]}=\mu_{\left(\operatorname{dim}\left(V_{1}+V_{2}\right)+\operatorname{dim}\left(V_{1} \cap V_{2}\right)\right)}^{[a]}$ and $v_{\left(\operatorname{dim}\left(V_{1}\right)+\left(\operatorname{dim}\left(V_{2}\right)\right.\right.}^{[b]}=$ $v_{\left(\operatorname{dim}\left(V_{1}+V_{2}\right)+\operatorname{dim}\left(V_{1} \cap V_{2}\right)\right)}^{[b]}$. Hence $\operatorname{dim}\left(V_{1}+V_{2}\right)+\operatorname{dim}\left(V_{1} \cap V_{2}\right)=\operatorname{dim}\left(V_{1}\right)+\operatorname{dim}\left(V_{2}\right)$. 


\section{Acknowledgements}

The authors express their sincere gratitude to the anonymous referees for their valuable and constructive suggestions which have improved the presentation.

The research of the first author is supported by UGC (University Grants Commission), India under Junior Research Fellowship in Science, Humanities and Social Sciences. The research of the second author is partially supported by the Special Assistance Programme (SAP) of UGC, New Delhi, India [Grant No. F 510/3/DRS-III/2015 (SAP-I)].

\section{References}

[1] Atanassov, K. T. (1986) Intuitionistic fuzzy sets, Fuzzy Sets and Systems, 20, 87-96.

[2] Atanassov, K. T. (1994) New operations defined over intuitionistic fuzzy sets, Fuzzy Sets and Systems, 61(2), 137-142.

[3] Atanassov, K. T. (1999) Intuitionistic Fuzzy Sets: Theory and Applications, Studies in Fuzziness and Soft Computing, Vol. 35, Springer Physica-Verlag, Heidelberg.

[4] Atanassov, K. T., (2012) On Intuitionistic Fuzzy Sets Theory, Studies in Fuzziness and Soft Computing, Vol. 283, Springer, Berlin.

[5] Atanassova, L. (2007) On intuitionistic fuzzy versions of L. Zadeh's extension principle, Notes on Intuitionistic Fuzzy Sets, 13(3), 33-36.

[6] Biswas, R. (1989) Intuitionistic fuzzy subgroups, Math. Forum, 10, 37-46.

[7] Biswas, R. (1997) On fuzzy sets and intuitionistic fuzzy sets, Notes on Intuitionistic Fuzzy Sets, 3(1), 3-11.

[8] Chen, W., \& Zhang, S. (2009) Intuitionistic fuzzy Lie sub-superalgebras and intuitionistic fuzzy ideals, Computers and Mathematics with Applications, 58, 1645-1661.

[9] Chiney, M., \& Samanta, S. K., Intuitionistic fuzzy basis of an intuitionistic fuzzy vector space, Notes on Intuitionistic Fuzzy Sets (Accepted).

[10] Coker, D. (1997) An introduction to intuitionistic fuzzy topological spaces, Fuzzy Sets and Systems, 88, 81-89.

[11] De, S. K., Biswas, R., \& Roy, A. R. (2001) An application of intuitionistic fuzzy sets in medical diagnostic, Fuzzy Sets and Systems, 117(2), 209-213.

[12] Ejegwa, P. A., Akubo, A. J., \& Joshua, O. M. (2014) Intuitionistic fuzzy set and its application in career determination via normalized euclidean distance method, European Scientific Journal, 10(15), 529-536. 
[13] Hur, K., Kang, H.W., \& Song, H. K. (2003) Intuitionistic fuzzy subgroups and subrings, Honam Math. J., 25, 19-41.

[14] Katsaras, A. K., \& Liu, D. B. (1977) Fuzzy vector spaces and fuzzy topological vector spaces, J. Math. Anal. Appl., 58, 135-146.

[15] Mohammed, M. J., \& Ataa, G. A. (2014) On Intuitionistic fuzzy topological vector space, Journal of College of Education for Pure Sciences, 4, 32-51.

[16] Mondal, K. K., \& Samanta, S. K. (2013) A study on intuitionistic fuzzy topological spaces, Notes on Intuitionistic Fuzzy Sets, 9(1), 1-32.

[17] Park, J. H. (2004) Intuitionstic fuzzy metric spaces, Chaos Solitons Fractals, 22, 10391046.

[18] Padmapriya, S., Uma, M. K., \& Roja, E. (2014) A study on intuitionistic fuzzy topological* groups, Annals of Fuzzy Mathematics and Informatics, 7(6), 991-1004.

[19] Pradhan, R., \& Pal, M. (2012) Intuitionistic fuzzy linear transformations, Annals of Pure and Applied Mathematics, 5(1), 57-68.

[20] Shi, F. G., \& Huang, C. E. (2010) Fuzzy bases and the fuzzy dimension of fuzzy vector spaces, Math. Commun., 15(2), 303-310.

[21] Szmidt, E., \& Kacprzyk, J. (1996) Intuitionistic fuzzy sets in group decision making, Notes on Intuitionistic Fuzzy Sets, 2(1), 11-14.

[22] Zadeh, L. A. (1965) Fuzzy sets, Information and Control, 8, 338-353. 